\title{
Design of test Platform for Robot Drilling and Cutting Force Simulation
}

\author{
Ying $\mathrm{Xu}^{1, \mathrm{a}^{*}}$, Renbin $\mathrm{He}^{1, \mathrm{~b}}$, Jianqiang Chen ${ }^{1, \mathrm{c}}$, Benshuang Zhang ${ }^{1, \mathrm{~d}}$, \\ Zhaolei $\mathrm{Li}^{1, \mathrm{e}}$ and Yanyu Deng ${ }^{1, \mathrm{f}}$
}

${ }^{1}$ School of Aircraft Engineering, Nanchang Hangkong University, Nangchang, JX, China, 330063

atn416ky@126.com.cn, b812740808@qq.com, 'c1099598361@qq.com, d631182510@qq.com

e956896308@qq.com, ${ }^{\dagger} 437102985 @ q q . c o m$

Corresponding author: tn416ky@126.com

Keywords: End-effector; dynamic simulation; cutting force; drilling vibrations; robots.

Abstract: In view of the requirements of aircraft parts automatic drilling processing, based on the analysis of the working process of the automatic drilling end-effector, through the steady-state design and the system structure modeling with CATIA, design a set of testing, drilling, pressure riveting, suck crumbs as one of the compound system of drilling end-effector. For building the structure design, virtual assembly and motion simulation for the integration of 3D virtual platform, it can realize the motion simulation of the multiple working procedures in the drilling operation. Adopted the large dynamic simulation software ADAMS and ABAQUS to analyze the cutting mechanism of robot drilling, the influence factors of the cutting force and clamping force of the robot are analyzed in the simulation, theoretical analysis of the accuracy error of process parameters to reduce the range of the robot system drilling. Finite element simulation study showed that dynamics simulation calculation can effectively solve the problem of process parameters settings for the robot system distributed, to provide theoretical reference for improving the drilling efficiency and assembly quality of aircraft components.

\section{Introduction}

Riveting connection is the main form of connection in aircraft assembly, the quality of the holes will influence the mechanical strength and assembly quality of aircraft. Robot automatic drilling system is a robot with multifunction drilling end executor and position calibration system to form a robot flexible drilling system[1]. The precise positioning and attitude adjustment of the end executor will be completed by robot, the rotation of the drill bit and feed will be complete by the end-effector[2], the machining process and position precision will be real-time measured by the monitor and calibration system.

Based on the system of precision drilling technology of robot, vibration of the robot have a significant impact on the accuracy of drilling, the basic cause of the vibration characteristics of the robot is the reaction force generated by the process of drilling[3]. The magnitude of the force is not only related to the work piece material, but also closely related to process parameters. In order to obtain the appropriate processing parameters, the cutting force of the impact machining quality must be simulated[4].

So the focus of the current article is to design a novel multi-drilling executer by focusing on the requirement of drilling vertical, design a set of testing, drilling, pressure riveting, suck crumbs as one of the compound system of drilling end-effector, to build the structure design, virtual assembly and motion simulation for the integration of 3D virtual platform, it can realize the motion simulation of the multiple working procedures in the drilling operation. We also presented the kinematics simulation model of the end-effector are outlined, and how the cutting force and clamping force change during the system drilling process. It provides drilling accuracy and parameters select in the process. 


\section{The structure design of the robot system platform}

Drilling making system constitutes the platform through designing the robot body structure with 6 degrees of freedom articulated robot series(shown in Fig.1) and adding a drilling end-effector (shown in Fig.2). In the process, joint type robots move the composite drilling units to the target riveting point, following with a series of process such as detecting, pressure riveting, drilling, suck crumbs completed by the composite drilling unit, which finally realizing automatic drilling features of the robot system[5].

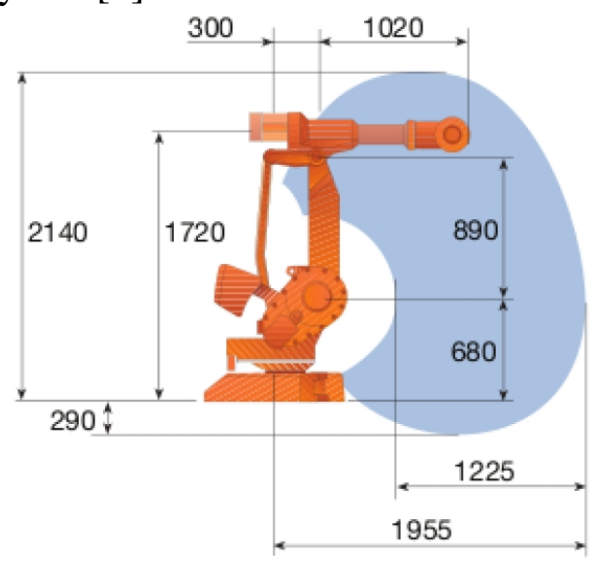

Fig.1. Industrial robot IRB4400/60 from ABB

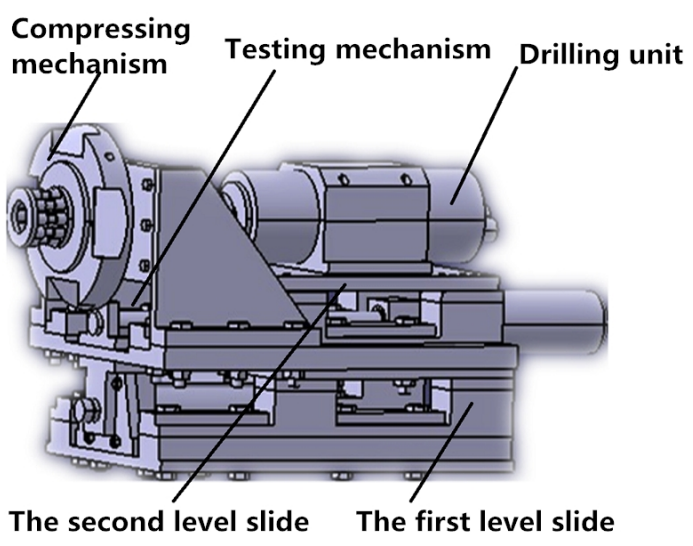

Fig.2. Structure of the end-effector

\section{Design of the drilling end-effector structure}

As shown in Fig.2, the design of the drilling end-effector is consists of five parts which are testing mechanism, compressing mechanism, drilling mechanism, chip removal mechanism and auxiliary mechanism. All parts are installed in two different levels on the slide, compressing mechanism chip removal mechanism and secondary slide component are installed in the first level slide, testing mechanism drilling mechanism are in the second. When compressing mechanism chip removal mechanism and secondary slide component are in the initial position the drilling end-effector is in original working state. When the control system sends the processing signal, the first levels slide pneumatic drive the second to the working position, the testing mechanism detect and adjust the tool's posture, compressing mechanism start, the pressure prism will be locked when it contact with the workpiece and pressured to a certain value; the motorized spindle of drilling mechanism rotate and do feeding movement, when the workpiece is completely drilled, motorized spindle reverse rotation and return to the initial position to complete the retract process; the piston rod returned, compressing mechanism is separated from the surface of the workpiece, and the pressure prism is returned to the initial position; the chip removal mechanism start and absorb chips to finish a drilling process, the drilling end-effector are connected to the joint robot by flange[6.7].

\section{Drilling force computing model of robot system platform}

In order to ensure the accuracy and improve the quality of drilling, it is necessary to analyze the internal relationship between the drilling thrust and technological parameters, drilling thrust is the main factor affecting the quality of drilling (shown in Fig.3) [6]. To better predict the drilling thrust under different technological parameters, the establishment of empirical formula about drilling thrust, drilling tool diameter, feed rate and cutting speed is required, the common forms of index formula are:

$$
F=C \cdot d^{X} \cdot f^{Y} \cdot n^{Z}
$$

In the formula, $F$ is the drilling thrust $(\mathrm{N}) ; f$ is the feed rate $(\mathrm{mm} / \mathrm{r}) ; n$ is the spindle speed $(\mathrm{r} / \mathrm{m})$; $d$ is the tool diameter (mm); the coefficient $C$ is related to the material and the thickness of the workpiece, the material and the shape of the drilling tool. According to the material and working 
conditions of drilling object, each value and coefficient of the formula are set.

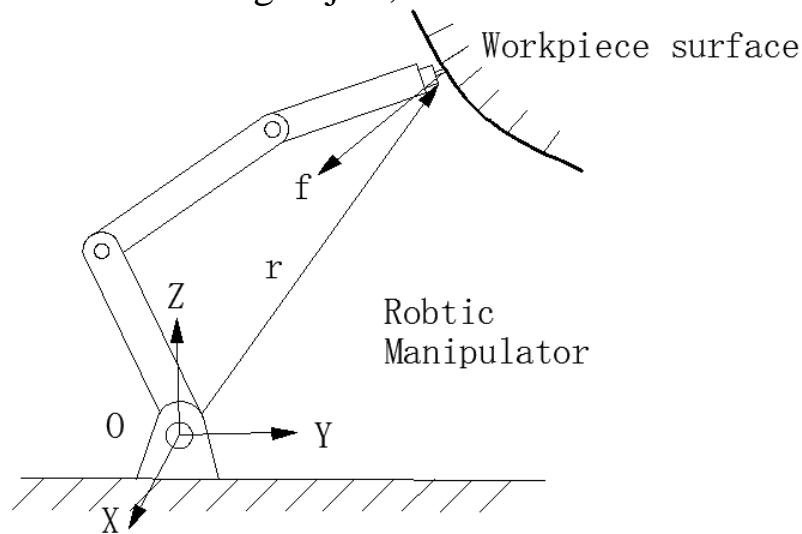

Fig 3. Drilling force computing model simulation

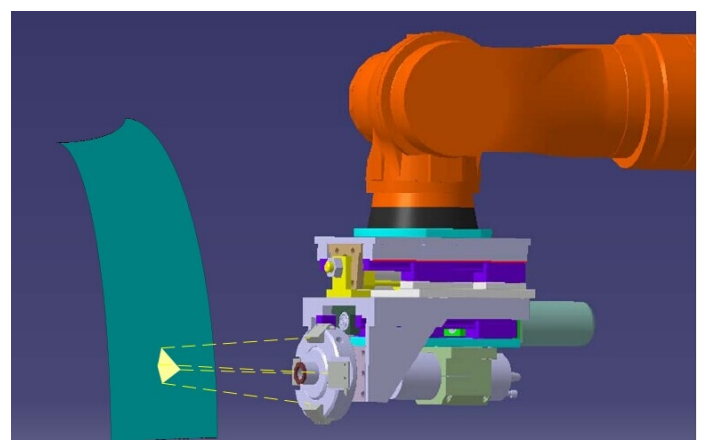

Fig.4. The working process of the drilling

\section{Robot drilling motion simulation}

Based on the dynamic simulation software ADMAS to construct a robot drilling virtual simulation platform and the designed end-effector is assembled with the ABB IRB4400/60 robot(shown in Fig.4), by two-way communication between robot and embedded controller digital I/O to have the function of double refers to zero, attitude adjustment, drilling, etc. This paper use the standard auger bit 30\#8", materials of hard alloy Y330. The workpiece model is a circular cylinder with diameter of $20 \mathrm{~mm}$ and height of $25 \mathrm{~mm}$, the material is Al2024. Adopt absolute meshing methods, using MATLAB software to solve the formula(1), the variation curve of drilling force can be obtained[8.9]

The main contents of the kinetic parameters of the drilling unit test are determine the parts clamping force(shown in Fig.5a), the cutting force(shown in Fig.5b), the feeding speed (shown in Fig.5c) and other parameters under the given driving and load conditions[9]. In the process of simulation, drilling unit movements takes place in the simulation cycle between $4 \sim 7 \mathrm{~s}$, which feed $2 \mathrm{~s}$ and $1 \mathrm{~s}$ contraction, and feed distance is $95 \mathrm{~mm}$. The driving function of the ball screw is:

step(time, 4,0,6,3420d)+step(time, 6,0,7,-3420d)

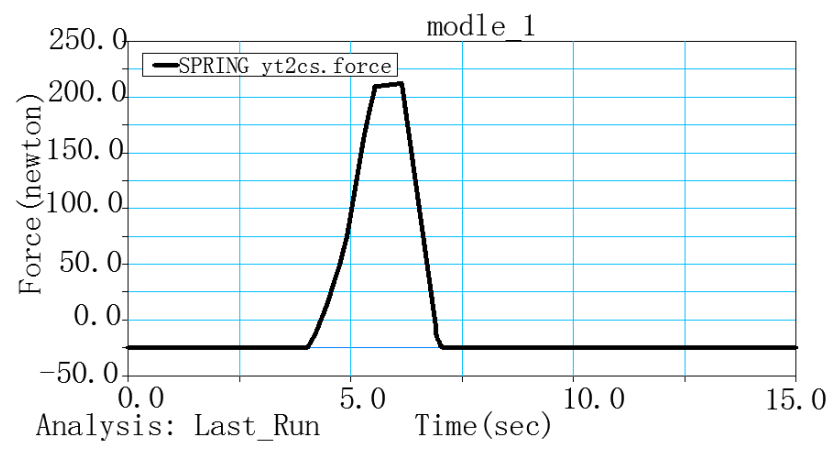

Fig.5a Drilling clamping force

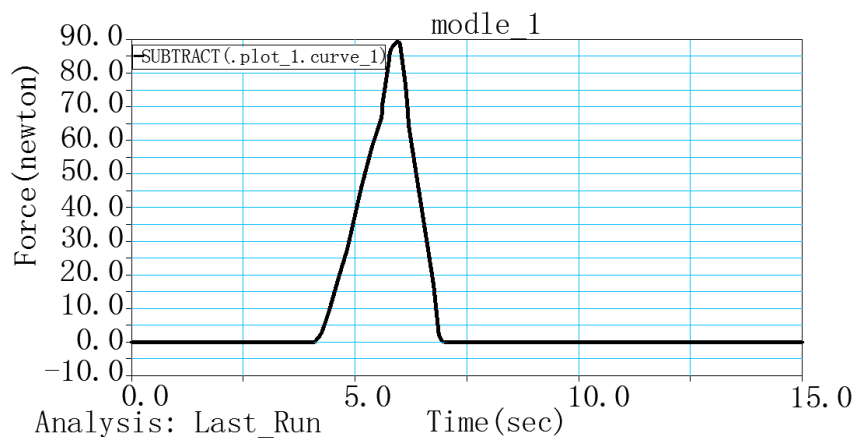

Fig.5b Drilling cutting force 


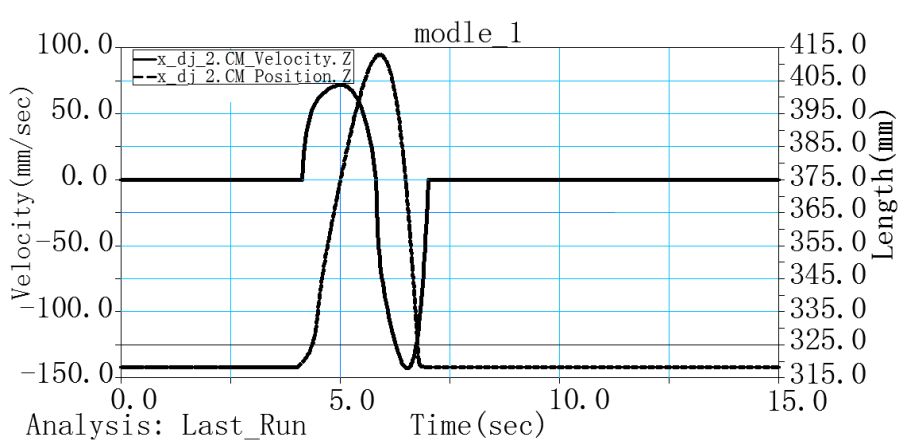

Fig.5c Bit feeding speed \& The displacement map pressure

As shown in Fig.5d, the parts came into contact with the clamping mechanism and clamping elements at $5.65 \mathrm{~s}$, clamping force is $210 \mathrm{~N}$, drilling feed force is $90 \mathrm{~N}$. At the same time can be seen from the figure 4 to 5 , there is a displacement of the clamping mechanism after contact with parts, which indicates that the fitting clearance between the two parts to be processed is eliminated, and the expected purpose is reached.

\section{Acknowledgements}

The authors would like to thank National Natural Science Foundation of China(Grant No. 51365042) and The Project Sponsored by the Scientific Research Foundation for the Returned Overseas Chinese Scholars, State Education Jiangxi (Grant No. DB201406147).

\section{Conclusion}

This paper presents on the technology analysis and motion simulation of the robot drilling process system, aiming to help understand the changes in the process and how the cutting force and clamping force change during the system drilling process. It provides the theoretical reference of vibration problems, improving drilling accuracy and parameters selected in the process. It provides a theoretical platform for the promotion of aircraft digital assembly through the steady-state design and terminal actuator system, having positive significance for changing installation and alignment, aircraft assembly rely on traditional manual operation mode, improving the assembly efficiency and drilling quality and laying a foundation for the next step of the terminal actuator system prototype development.

\section{References}

[1] Ch.K. Wang, P.J. Yuan, T.M. Wang, Q.S. Wang, H.J Tan, D.D. Chen, T. Lai, The Study of Drilling and Countersink Technology in Robot Drilling End-effector. IEEE 9th Conference on Industrial Electronics and Applications (ICIEA)2014, pp. 1859-1863.

[2] L.D. Marcos,J. J. Gil, A mechatronic bone drilling tool, Mechatronics,2( 2012) 1060-1066.

[3] M. Gong, P. Yuan, T. Miao and R. Zhang, Intelligent verticality-adjustment method of end-effector in aeronautical drilling robot. Journal of Beijing University of Aeronautics and Astronautics, 10 (2012) 1400-1404.

[4] L. Wang and T.N. Feng, Application of Digital Automatic Drill-Riveting Technology in Aircraft Manufacture. Aeronautical Manufacturing Technology, 5 (2008) 42-45.

[5] X.P. Hu, Y. Xu, W.F. Zheng and H. Xu, Design and Dynamic Analysis of Automatic Aircraft Assembly Robot. Manufacturing automation, In Chinese, 7 ( 2009 ) ,25-29. 
[6] Baorui Du, Ziming Feng, Yanbin Yao and Shusheng Bi, Robot Drilling System for Automatic Drilling of Aircraft. Aeronautical Manufacturing Technology, 2(2010) 47-50.

[7] Y. Xu, L. Wang and Y. Zhao, Design of Riveting Deformation Detective Element Based on Displacement Sensor. Advanced Materials Research, 503-504(2012)1562-1565.

[8] J. Wang, H. Liu, W. Tian, S. Wan, Y. Liu and D. Li, Design of drilling end-effector for aircraft automatic assembly. Journal of Nanjing University of Aeronautics \& Astronautics, 4(2012) 19-22.

[9] Ying Xu, Liang Wang, Yu Zhao, Design of Riveting Deformation Detective Element Based on Displacement Sensor. Advanced Materials Research, 503-504(2012) 1562-1565. 